\title{
A general form of relative recursion
}

\author{
Jaap van Oosten \\ Department of Mathematics \\ Utrecht University \\ P.O.Box 80.010, 3508 TA Utrecht, The Netherlands \\ jvoosten@math.uu.nl
}

July 15, 2018

\begin{abstract}
The purpose of this note is to observe a generalization of the concept "computable in..." to arbitrary partial combinatory algebras. For every partial combinatory algebra (pca) $A$ and every partial endofunction on $A$, a pca $A[f]$ is constructed such that in $A[f]$, the function $f$ is representable by an element; a universal property of the construction is formulated in terms of Longley's 2-category of pcas and decidable applicative morphisms.

It is proved that there is always a geometric inclusion from the realizability topos on $A[f]$ into the one on $A$, and that there is a meaningful preorder on the partial endofunctions on $A$ which generalizes Turing reducibility.
\end{abstract}

AMS Subject Classification (2000): 03B40,68N18

\section{Introduction}

In [5], John Longley defined a 2-category of partial combinatory algebras (see 0.1.1 and 0.1.2 for definitions). The morphisms are different from what one might expect: rather that 'algebraic' maps, they are more like simulations (of one world of computation in another). Accordingly, a morphism from $A$ to $B$ is a total relation between the underlying sets.

Longley's definition made a lot of sense since there are nice functorial connections between pcas and their corresponding realizability categories (realizability toposes and categories of assemblies).

However, the 2-category has not been studied in great detail. It does not appear to have a lot of categorical structure, and not much is known. 
Fundamental questions, such as: which properties of partial combinatory algebras are stable under isomorphism, or equivalence?, have not been answered (indeed, such questions have hardly been posed).

In this paper, I present a simple construction which is available in this category: adjoin a partial function. That is, given a pca $A$ and a partial endofunction $f$ on $A$, construct a pca $A[f]$ in which the function $f$ is 'computable'. $A[f]$ should, of course, possess a universal property, and this property is formulated with respect to what Longley calls 'decidable' morphisms.

Characteristically for the non-algebraic flavour of the 2-category, $A[f]$ is not constructed by adding elements, but by modifying the application function. We obtain results generalizing the situation of computing relative to an oracle: a preorder, similar to (and generalizing) Turing reducibility, can be defined on the partial endofunctions on $A$; and there is always a geometric inclusion from the realizability topos on $A[f]$ into the one on $A$.

It is also a surprising corollary of this work that every total pca is isomorphic to a nontotal one.

\subsection{Basic notions and notations}

\subsubsection{Partial combinatory algebras}

A partial combinatory algebra (pca) is a set $A$ together with a partial function $A \times A \rightarrow A$ called application, which satisfies a few conditions. We write the application as $(a, b) \mapsto a b$ or $a \cdot b$. $a b \downarrow$ means that the application $a b$ is defined. When dealing with compound terms like $(a c)(b c)$, the definedness of the term is meant to imply the definedness of every subterm. For terms $t$ and $s$, the notation $t \simeq s$ means that $t$ is defined exactly when $s$ is; and that they deonte the same element when defined. $t=s$ will mean $t \simeq s$ and $t \downarrow$. As usual, we associate to the left, that is: $a b c$ means $(a b) c$. Elements of $A$ are usually called combinators.

With these conventions, $(A, \cdot)$ is a pca iff there are combinators $K$ and $S$ in $A$ satisfying, for all $a, b, c \in A$ :

- $K a b=a$

- $S a b \downarrow$

- $S a b c \simeq a c(b c)$

For a careful account of the theory of pcas, see [1] or [5]. We recall a few properties. 
In a pca $A$ there is a choice of Booleans $\top$ and $\perp$, and a 'definition by cases' combinator $C$ such that for all $a, b \in A, C \top a b=a$ and $C \perp a b=b ; C$ is pronounced (and written) as If. . then... else....

In $A$ there is a choice of elements $\bar{n}$ for every natural number $n$, such that for every partial recursive function $F$ of $k$ variables there is a combinator $a_{F}$ such that for every $k$-tuple $\left(n_{1}, \ldots, n_{k}\right), a_{F} \overline{n_{1}} \ldots \overline{n_{k}} \downarrow$ precisely when $F\left(n_{1}, \ldots, n_{k}\right)$ is defined, and $a_{F} \overline{n_{1}} \ldots \overline{n_{k}}=\overline{F\left(n_{1}, \ldots, n_{k}\right)}$ if this is the case. There is a coding of finite sequences of elements of $A$, together with combinators which allow us to manipulate them: if we write $\left[u_{0}, \ldots, u_{n-1}\right]$ for the code of the sequence $\left(u_{0}, \ldots, u_{n-1}\right)$, there is a combinator Ih which gives the length of the coded sequence (i.e. $\operatorname{lh}\left[u_{0}, \ldots, u_{n-1}\right]=\bar{n}$ ), there are combinators picking the $i$-th element of the coded sequence (we simply write $u_{i}$ for its effect) and a concatenation operator; we write $\left[u_{0}, \ldots u_{n-1}\right] *\left[v_{0}, \ldots, v_{m-1}\right]$ for the effect of this last combinator.

All these facts follows from the existence, in $A$, of a combinator for primitive recursion. Moreover, in every pca $A$ there is a fixpoint combinator $Y$ satisfying: $Y f \downarrow$ for all $f \in A$, and $Y f a \simeq f(Y f) a$. We shall refer to this fact as 'the recursion theorem in $A$ '.

Every pca $A$ is 'combinatory complete': for every term $t$ (constructed from variables, constants from $A$, and the application function) and every sequence of variables $x_{1}, \ldots x_{n+1}$ which contains all variables in $t$, there is an element $\Lambda^{*} x_{1} \cdots x_{n+1} . t$ in $A$ which satisfies for all $a_{1}, \ldots a_{n+1}$ in $A$ :

- $\left(\Lambda^{*} x_{1} \cdots x_{n+1} . t\right) a_{1} \cdots a_{n} \downarrow$

- $\left(\Lambda^{*} x_{1} \cdots x_{n+1} . t\right) a_{1} \cdots a_{n+1} \simeq t\left(a_{1}, \ldots, a_{n+1}\right)$

\subsubsection{Longley's 2-category of pcas; assemblies; decidable maps}

The following definition is due to John Longley ([5]).

Definition 0.1 Let $A$ and $B$ be pcas. An applicative morphism from $A$ to $B$ is a function $\gamma$ from $A$ to the set $\mathcal{P}^{*}(B)$ of nonempty subsets of $B$, such that there exists an element $r \in B$ with the property that if $a a^{\prime} \downarrow$ in $A$, $b \in \gamma(a)$ and $b^{\prime} \in \gamma\left(a^{\prime}\right)$, then $r b b^{\prime} \downarrow$ and $r b b^{\prime} \in \gamma\left(a a^{\prime}\right)$. The element $r$ is said to be a realizer for $\gamma$.

Given two applicative morphisms $\gamma: A \rightarrow B$ and $\delta: B \rightarrow C$, the composition $\delta \gamma: A \rightarrow C$ is the function $a \mapsto \bigcup_{b \in \gamma(a)} \delta(b)$ from $A$ to $\mathcal{P}^{*}(C)$. It is easy, using combinatory completeness, to find a realizer for $\delta \gamma$ in terms of realizers for $\gamma$ and $\delta$. 
This composition is evidently associative and has identities $a \mapsto\{a\}$, so we have a category of pcas.

This category is preorder-enriched: given two applicative morphisms $\gamma, \delta: A \rightarrow B$, we say $\gamma \preceq \delta$ if there is an $s \in B$ such that for all $a \in A$ and all $b \in \gamma(a), s b \in \delta(a)$. We say that $\gamma$ and $\delta$ are isomorphic if $\gamma \preceq \delta$ and $\delta \preceq \gamma$ both hold.

Two pcas are equivalent if there are $\gamma: A \rightarrow B$ and $\delta: B \rightarrow A$ such that both composites are isomorphic to identities.

An assembly on a pca $A$ is a set $X$ together with a map $E_{X}: X \rightarrow \mathcal{P}^{*}(A)$. If $\left(X, E_{X}\right)$ and $\left(Y, E_{Y}\right)$ are assemblies on $A$, a map of assemblies is a function $f: X \rightarrow Y$ such that there is an element $r \in A$ such that for all $x \in X$ and all $a \in E_{X}(x), r a \downarrow$ and $r a \in E_{Y}(f(x))$. One says that the element $r$ tracks the function $f$. Assemblies on $A$ and maps of assemblies form a category $\operatorname{Asm}(A)$. This category is regular and comes equipped with an adjunction to the category of Sets: the forgetful (or global sections) functor $\Gamma: \operatorname{Asm}(A) \rightarrow$ Set is left adjoint to the functor $\nabla: \operatorname{Set} \rightarrow \operatorname{Asm}(A)$ which sends a set $X$ to the pair $\left(X, E_{X}\right)$ where $E_{X}(x)=A$ for all $x \in X$.

An important justification for definition 0.1 is the following theorem by Longley: every applicative morphism $\gamma: A \rightarrow B$ determines a regular functor $\gamma^{*}: \operatorname{Asm}(A) \rightarrow \operatorname{Asm}(B)$ which commutes with the functors $\Gamma$; conversely, every such functor is induced by an applicative morphism which is unique up to isomorphism.

Note, that $\gamma: A \rightarrow B$ establishes $A$ as an assembly on $B$.

Definition 0.2 A morphism $\gamma: A \rightarrow B$ is decidable if there is an element $d \in B$ (the decider for $\gamma$ ) such that if $\top_{A}, \perp_{A}$ are the Booleans in $A$ and $\top_{B}, \perp_{B}$ the Booleans in $B$, for every $b \in \gamma\left(\top_{A}\right)$ we have $d b=\top_{B}$ and for every $b \in \gamma\left(\perp_{A}\right), d b=\perp_{B}$.

In 5], Longley proved

Proposition 0.3 An applicative morphism $\gamma: A \rightarrow B$ is decidable if and only if the corresponding functor $\gamma^{*}: \operatorname{Asm}(A) \rightarrow \operatorname{Asm}(B)$ preserves finite coproducts. Moreover this is equivalent to: $\gamma^{*}$ preserves the natural numbers object.

Corollary 0.4 If $\delta=\gamma \zeta$ is a commutative triangle of applicative morphisms such that $\delta$ and $\zeta$ are decidable, then so is $\gamma$. 


\section{Definition of $A[f]$ and basic properties}

Definition 1.1 Let $\gamma: A \rightarrow B$ be an applicative morphism of pcas and $f: A \rightarrow A$ a partial function. We say that $f$ is representable w.r.t. $\gamma$ if there is an element $r_{f} \in B$ such that for every $a \in \operatorname{dom}(f)$ and every $b \in \gamma(a), r_{f} b \downarrow$ and $r_{f} b \in \gamma(f(a))$. We say that $f$ is representable in $A$ if $f$ is representable w.r.t. the identity morphism on $A$.

The representability of $f$ with respect to $\gamma$ can also be seen as follows: let $(\operatorname{dom}(f), \gamma)$ be the regular sub-assembly of $(A, \gamma)$ (as assemblies on $B)$. Then $f$ is representable with respect to $\gamma$ if and only if $f$ is a map of assemblies: $(\operatorname{dom}(f), \gamma) \rightarrow(A, \gamma)$.

Theorem 1.2 For every pca $A$ and every partial endofunction $f$ on $A$ there exist a pca $A[f]$ and a decidable applicative morphism $\iota_{f}: A \rightarrow A[f]$ with the following properties:

i) $f$ is representable w.r.t. $\iota_{f}$;

ii) for every decidable applicative morphism $\gamma: A \rightarrow B$ such that $f$ is representable w.r.t. $\gamma$, there is a decidable applicative morphism $\gamma_{f}$ : $A[f] \rightarrow B$ such that $\gamma_{f} \iota_{f}=\gamma$, and $\gamma_{f}$ is unique with this property. Moreover, if $\delta: A[f] \rightarrow B$ is such that $\delta \iota_{f} \cong \gamma$, then $\delta \cong \gamma_{f}$

Proof. For the construction of $A[f]$, let's agree on some notation for codes of finite sequences: if $u=\left[u_{o}, \ldots, u_{n-1}\right]$ and $i<n, u^{<i}$ denotes $\left[u_{0}, \ldots, u_{i-1}\right]$ and $u^{\geq i}$ denotes $\left[u_{i}, \ldots, u_{n-1}\right]$; for $i \leq j<n,{ }^{i \leq} u^{<j}$ denotes $\left[u_{i}, \ldots, u_{j-1}\right]$. Let $p, p_{0}, p_{1}$ be pairing and projection combinators in $A$, i.e. satisfying for all $a, b \in A: p_{0}(p a b)=a$ and $p_{1}(p a b)=b$. Let Not be a combinator such that $\operatorname{Not} \top=\perp$ and Not $\perp=\top$.

The underlying set of $A[f]$ will be $A$. We define a new application . $f$ on $A$ as follows. For $a, b \in A$, an $f$-dialogue between $a$ and $b$ is a code of a sequence $u=\left[u_{0}, \ldots, u_{n-1}\right]$ such that for all $i<n$ there is a $v_{i} \in A$ such that

$$
a \cdot\left([b] * u^{<i}\right)=p \perp v_{i} \text { and } f\left(v_{i}\right)=u_{i}
$$

We say that $a \cdot{ }^{f} b$ is defined with value $c$, if there is an $f$-dialogue $u$ between $a$ and $b$ such that

$$
a \cdot([b] * u)=p \top c
$$

We show first, that $\left(A,{ }^{f}\right)$ is a pca.

Let $K_{f}=\Lambda^{*} x \cdot p \top\left(\Lambda^{*} y \cdot p \top x_{0}\right)$. Then clearly $K_{f} \cdot{ }^{f} a=\Lambda^{*} y \cdot p \top a$ for all $a \in A$, so $\left(K_{f} .{ }^{f} a\right) \cdot{ }^{f} b=a$ for all $a, b \in A$. 
For the combinator $S_{f}$, by primitive recursion it is possible to construct a term $t(x, y)$ of $A$ such that for all $u$, the application $t(x, y) \cdot u$ is given by the following instructions:

$t(x, y) \cdot u=$

$x u$ if $\forall i \leq \operatorname{lh} u \operatorname{Not}\left(p_{0}\left(x u^{<i}\right)\right)$.

If $i$ is minimal such that $p_{0}\left(x u^{<i}\right)$, let $\alpha=p_{1}\left(x u^{<i}\right)$ and output $y\left(\left[u_{0}\right] * u^{\geq i}\right)$ if $\forall j\left(i \leq j<\operatorname{lh} u \rightarrow \operatorname{Not} p_{0}\left(y\left(\left[u_{0}\right] *^{i \leq} u^{<j}\right)\right)\right.$.

If $j$ is minimal such that $p_{0}\left(y\left(\left[u_{0}\right] *^{i \leq} u^{<j}\right)\right)$, let $\beta=p_{1}\left(y\left(\left[u_{0}\right] *^{i \leq}\right.\right.$ $\left.\left.u^{<j}\right)\right)$ and output $\alpha\left([\beta] * u^{\geq j}\right)$ if $\forall k\left(j \leq k<\operatorname{lh} u \rightarrow \operatorname{Not}\left(p_{0}\left(\alpha\left([\beta] *^{j \leq}\right.\right.\right.\right.$ $\left.\left.\left.u^{<k}\right)\right)\right)$ ).

If $k$ is minimal such that $\left(p_{0}\left(\alpha\left([\beta] *^{j \leq} u^{<k}\right)\right)\right)$, output $\left(p_{1}\left(\alpha\left([\beta] *^{j \leq}\right.\right.\right.$ $\left.\left.\left.u^{<k}\right)\right)\right)$.

Note, that $t(a, b) \cdot{ }^{f} c \simeq\left(a \cdot{ }^{f} c\right) \cdot{ }^{f}\left(b \cdot{ }^{f} c\right)$ for all $a, b, c$. Therefore, let

$$
S_{f}=\Lambda^{*} x \cdot p \top\left(\Lambda^{*} y \cdot p \top t\left(x_{0}, y_{0}\right)\right)
$$

Then $\left(S_{f} .{ }^{f} a\right) \cdot{ }^{f} b=t(a, b)$ for all $a$ and $b$. This establishes $A[f]$ as a pca.

Note that the combinators $K_{f}$ and $S_{f}$ don't really depend on $f$. This is analogous to the fact that for a coding of Turing machine computations with oracle $U$, the $S_{n}^{m}$-functions are primitive recursive, and do not depend on $U$.

The map $\iota_{f}: A \rightarrow A[f]$ given by $a \mapsto\{a\}$ is an applicative morphism $A \rightarrow A[f]$. Indeed, if $a b=c$ then $\left(\Lambda^{*} x \cdot p \top\left(a x_{0}\right)\right) \cdot{ }^{f} b=c$; so if $r=\Lambda^{*} y x . p \top\left(y_{0} x_{0}\right)$ then $r$ realizes $\iota_{f}$.

The decidability of $\iota_{f}$ is left to the reader.

For the universal property, suppose $\gamma: A \rightarrow B$ is a decidable applicative morphism which is realized by $r$ and let $d$ be a decider for $\gamma$. Moreover suppose that $\bar{f}$ represents $f$ w.r.t. $\gamma$.

Let $\pi_{0}, \pi_{1} \in B$ be such that if $b \in \gamma(a)$ then $\pi_{i} b \in \gamma\left(p_{i} a\right)$. Similarly, let $C$ and $C^{\prime}$ in $B$ be such that if $b \in \gamma(a)$ and $v \in \gamma(u)$ then $C b v \in \gamma([a] * u)$ and $C^{\prime} b v \in \gamma(u *[a])$.

Now use the recursion theorem in $B$ to find an element $U$ such that for all $b, b^{\prime}, v$ :

$$
\begin{aligned}
U b b^{\prime} v \simeq & \text { If } d\left(\pi_{0}\left(r b\left(C b^{\prime} v\right)\right)\right) \\
& \text { then } \pi_{1}\left(r b\left(C b^{\prime} v\right)\right) \\
& \text { else } U b b^{\prime}\left(C^{\prime}\left(\bar{f}\left(\pi_{1}\left(r b\left(C b^{\prime} v\right)\right)\right)\right) v\right)
\end{aligned}
$$

The reader can check the following: suppose $u$ is an $f$-dialogue between $a$ and $a^{\prime}$ in $A, b \in \gamma(a), b^{\prime} \in \gamma\left(a^{\prime}\right), i<\operatorname{lh} u, v \in \gamma\left(u^{<i}\right)$ and $w=C^{\prime}\left(\bar{f}\left(\pi_{1}\left(r b\left(C b^{\prime} v\right)\right)\right)\right) v$. 
Then $w \in \gamma\left(u^{\leq i}\right)$ and $U b b^{\prime} v=U b b^{\prime} w$. Furthermore, if $u$ is such that $a\left(\left[a^{\prime}\right] * u\right)=p \top c$, then $U b b^{\prime} v \in \gamma(c)$.

Therefore, choose $e \in \gamma([])$ and let

$$
\rho=\Lambda^{*} x x^{\prime} . U x x^{\prime} e
$$

Then $\rho$ realizes $\gamma$ as applicative morphism: $A[f] \rightarrow B$. We denote this last morphism by $\gamma_{f}$.

Obviously, the diagram

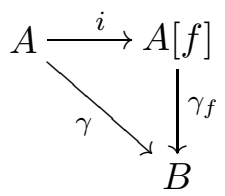

commutes on the nose. Moreover, since $\iota_{f}(a)=\{a\}$, if $\delta: A[f] \rightarrow B$ were such that $\delta \iota \cong \gamma_{f} \iota$, then $\delta \cong \gamma_{f}$. So $\gamma_{f}$ is unique with respect to the property that the diagram commutes on the nose, and essentially unique with respect to the property that it commutes up to isomorphism. The decidability of $\gamma_{f}$ is a direct consequence of Corollary 0.4 and can also be verified directly.

\section{Corollary 1.3}

i) If $f$ is representable in $A$, then $A$ and $A[f]$ are isomorphic pcas.

ii) If $f$ and $g$ are two partial endofunctions on $A$, the pcas $A[f][g]$ and $A[g][f]$ are isomorphic; we may therefore write $A[f, g]$.

iii) If $\mathcal{K}_{1}$ denotes Kleene's pca of partial recursive application, $f: \mathbb{N} \rightarrow \mathbb{N}$ is a partial function and $\mathcal{K}_{1}^{f}$ is the pca of partial recursive application with an oracle for $f$, then $\mathcal{K}_{1}^{f}$ is isomorphic to $\mathcal{K}_{1}[f]$.

iv) There exists a nontotal pca which is isomorphic to a total pca.

Proof. The first two statements are immediate from the uniqueness statement in theorem 1.2. The third statement is easy. Finally, the fourth statement follows from the fact that $A[f]$ is never total (the element $a=\Lambda^{*} x \cdot p \perp \perp$ is such that $a \cdot{ }^{f} b$ is never defined), so if $A$ is total and $f$ is representable in $A$, then $A \cong A[f]$ by i).

Example In [7, a total combinatory algebra $\mathcal{B}$ of partial functions on $\mathbb{N}$ is defined, and it is proved that the representable functions are those functions which are continuous for the Scott topology and satisfy some "sequentiality" 
condition. One might consider what happens if a "parallel" function is adjoined to this: e.g. let $F: \mathcal{B} \rightarrow \mathcal{B}$ be the function such that for all $\alpha \in \mathcal{B}$, $F(\alpha)(0)=0$ if and only if $0 \in \operatorname{dom}(\alpha)$ or $1 \in \operatorname{dom}(\alpha)$ (and undefined else), and $F(\alpha)(n)$ is undefined for all $n>0$. What would the representable functions of $\mathcal{B}[F]$ be? My conjecture would be that these are exactly all Scott-continuous functions on $\mathcal{B}$.

\section{Remarks}

1. The construction of $A[f]$ induces a preorder on the set of partial endofunctions of $A$, which generalizes Turing degrees: let $f \leq_{A} g$ if and only if $f$ is representable in $A[g]$ (with respect to $\iota_{g}$ ). Since the diagram

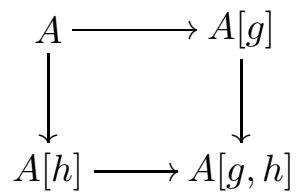

commutes, it is easy to see that $\leq_{A}$ is a transitive relation (it is reflexive by $1.2(i)$ ).

2. There is a universal solution to the problem of "making $A$ decidable"; adjoin a function $f$ to $A$ where

$$
f(x)= \begin{cases}\top & \text { if } p_{0} x=p_{1} x \\ \perp & \text { else }\end{cases}
$$

3. This seems to be a good point to correct a claim made in [2], lemma 5.4. It is claimed that no total pca can be equivalent to a pca $A$ in which there is an element $z$ such that for all $x, z x \downarrow$ and $z x \neq x$. However, this is established only if "equivalent" is replaced by "isomorphic". Therefore the original claim remains an open problem. Another open problem, as far as I know, is: give an example of two pcas which are equivalent, but not isomorphic.

\section{A geometric inclusion of realizability toposes}

The construction of $A[f]$ generalizes another aspect of relative recursion, known from the theory of realizability toposes. It is well known that for every pca $A$ there exists a realizability topos $\mathrm{RT}(A)$. The best studied example is $\mathrm{RT}\left(\mathcal{K}_{1}\right)$, the effective topos(4]). In [4] and [6] it is explained that $\operatorname{RT}\left(\mathcal{K}_{1}^{f}\right)$ 
is a subtopos of $\mathrm{RT}\left(\mathcal{K}_{1}\right)$, in the topos-theoretic sense. Here we shall see that this generalizes to geometric inclusions $\mathrm{RT}(A[f]) \rightarrow \mathrm{RT}(A)$.

In [2], the authors analyze a generalization of Longley's 2-category of pcas, and characterize which applicative morphisms give rise to geometric morphisms between realizability toposes. The key concept is that of a computationally dense morphism. Unfortunately, the definition given in l.c. is not quite adequate; see also [3. I state the correct definition here for pcas.

Definition 2.1 Suppose that $F: A \rightarrow B$ is a function between pcas such that the map $a \mapsto\{F(a)\}$ is an applicative morphism. $F$ is computationally dense if there is an $m \in B$ with the property that for every $b \in B$ one can find an $a \in A$ such that for all $a^{\prime} \in A$ :

$$
\text { If } b F\left(a^{\prime}\right) \downarrow \text { in } B \text {, then } a a^{\prime} \downarrow \text { in } A \text {, and } m F\left(a a^{\prime}\right)=b F\left(a^{\prime}\right)
$$

Let $P(A)$ and $P(B)$ denote the realizability triposes on $A$ and $B$. Then in [2] it is shown that the map of indexed preorders induced by $F^{*}$ (where $F^{*}: \mathcal{P}(A) \rightarrow \mathcal{P}(B)$ sends $\alpha$ to $\left.F[\alpha]\right)$ has an indexed right adjoint if and only if $F$ is computationally dense.

In that case, the right adjoint is induced by the map $\hat{F}: \mathcal{P}(B) \rightarrow \mathcal{P}(A)$, given by

$$
\hat{F}(\beta)=\{a \in A \mid m F(a) \in \beta\}
$$

where $m \in B$ witnesses the computational density of $F$.

It is easily verified then, that if $F$ is computationally dense and $m$ is as in definition 2.1. then the geometric morphism $\left(\hat{F}, F^{*}\right)$ is an inclusion precisely when the following condition holds:

(in) There is a $c \in B$ such that for every $b \in B$ there is an $a \in A$ such that $c b=F(a)$ and $m(c b)=b$

Proposition 2.2 The identity function $A \rightarrow A[f]$ is computationally dense and satisfies the condition (in).

Proof. This is quite simple. Let $m$ be an element of $A$ such that for every $y \in A$ and every code of a sequence $v, m([y] * v) \simeq y v$.

Given $b \in A$, let $a \in A$ be such that for all $a^{\prime} \in A, a a^{\prime} \simeq \Lambda^{*} v \cdot b\left(\left[a^{\prime}\right] * v\right)$. Then $a a^{\prime}$ is always defined. Moreover,

$$
m\left(\left[a a^{\prime}\right] * v\right) \simeq\left(a a^{\prime}\right) v \simeq b\left(\left[a^{\prime}\right] * v\right)
$$

It follows that $m \cdot{ }^{f}\left(a a^{\prime}\right) \simeq b \cdot{ }^{f} a^{\prime}$ in $A[f]$. This proves that the identity function is computationally dense. 
Moreover, if $c=\Lambda^{*} x \cdot p \top\left(\Lambda^{*} v \cdot p \top v_{0}\right)$ then for all $a, c[a]=p \top(\Lambda v \cdot p \top a)$; hence $c . f^{f} a=\Lambda^{*} v \cdot p \top a$ and

$$
m\left(\left[c \cdot{ }^{f} a\right]\right)=\left(c \cdot{ }^{f} a\right)[]=p \top a
$$

so $m \cdot f\left(c . f^{f} a\right)=a$, which proves (in).

\section{References}

[1] I. Bethke,Notes on Partial Combinatory Algebras, Ph.D. thesis, Universiteit van Amsterdam,1988

[2] P. Hofstra, P. and J. van Oosten, Ordered partial combinatory algebras, Math. Proc. Camb. Phil. Soc.134 (2003), 445-463

[3] P. Hofstra, P. and J. van Oosten, Erratum to 'Ordered partial combinatory algebras', (2003), available at http://www.math.uu.nl/people/jvoosten/realizability/erratum.ps

[4] J.M.E. Hyland, The effective topos, in: A.S. Troelstra and D. Van Dalen (eds), The L.E.J. Brouwer Centenary Symposium, North Holland Publishing Company 1982, pp. $165-216$

[5] J. Longley, Realizability Toposes and Language Semantics, Ph.D. thesis, Edinburgh University 1995

[6] W.K.-S. Phoa, Relative Computability in the Effective Topos, Math. Proc. Camb. Phil. Soc. 106 (1989), 419-422

[7] J. van Oosten, A Combinatory Algebra for Sequential Functionals of Finite Type in: Cooper, S.B. and Truss, J.K. (eds), Models and Computability, Cambridge University Press 1999, pp. 389-406] 